\title{
SECOND-ORDER DIFFERENTIAL EQUATIONS WITH FRACTIONAL TRANSITION POINTS(1)
}

BY

\author{
F. W. J. OLVER
}

ABstract. An investigation is made of the differential equation

$$
d^{2} w / d x^{2}=\left\{u^{2}\left(x-x_{0}\right)^{\lambda} f(u, x)+g(u, x) /\left(x-x_{0}\right)^{2}\right\} w,
$$

in which $u$ is a large real (or complex) parameter, $\lambda$ is a real constant such that $\lambda>-2, x$ is a real (or complex) variable, and $f(u, x)$ and $g(u, x)$ are continuous (or analytic) functions of $x$ in a real interval (or complex domain) containing $x_{0}$. The interval (or domain) need not be bounded. Previous results of Langer and Riekstiņ giving approximate solutions in terms of Bessel functions of order $1 /(\lambda+2)$ are extended and error bounds supplied.

1. Introduction and summary. A differential equation of the form

$$
d^{2} w / d x^{2}=\left\{u^{2} f(x)+g(x)\right\} w,
$$

in which $u$ is a large parameter and the functions $f(x)$ and $g(x)$ are independent of $x$, is said to have a simple transition point, or simple turning point, at $x=x_{0}$ if $f\left(x_{0}\right)=0$ and $f^{\prime}\left(x_{0}\right) \neq 0$. More generally, if $f(x)$ has a zero at $x_{0}$ of order $m$, then equation (1.01) is said to have a transition point of order $m$ at $x=x_{0}$.

The foregoing definition may be extended to cases in which

$$
f(x)=\left(x-x_{0}\right)^{\lambda} h(x),
$$

where $h(x)$ is finite and nonvanishing at $x_{0}$, and the constant $\lambda$ is an unrestricted real number, usually to be regarded as fixed. In these circumstances we may say that equation (1.01) has a transition point of fractional order $\lambda$ at $x_{0}$. The problem of constructing asymptotic solutions of $(1.01)$ for large $u$ which are uniformly valid in a region containing $x_{0}$ was first solved for arbitrary values of $\lambda$ by Langer [1], [2], [3]. In [1] Langer made the following

Received by the editors January 28, 1975 and, in revised form, November 25, 1975.

AMS (MOS) subject classifications (1970). Primary 34E20; Secondary 33A40.

Key words and phrases. Asymptotic analysis, Bessel functions, error analysis, linear differential equations, transition point, turning point.

(1) This research was supported by the U. S. Army Research Office, Durham under Contract DA-ARO-D-31-124-73-G204, and the National Science Foundation under Grant GP 32841X. 
assumptions: $\lambda \geqslant 0$; the variable $x$ ranges over a bounded or unbounded real interval containing the fractional transition point $x_{0}$; the function $g(x)$ is bounded as $x \rightarrow x_{0}$; and the large parameter $u$ is real or complex. In [2] these results were extended to the complex $x$-plane. In the third paper [3] he made further extensions to permit $\lambda>-2$, and $g(x)$ to possess a simple or double pole at $x_{0}$. In each case Langer constructed approximations for the solutions in terms of Bessel functions of fixed order, accompanied by asymptotic estimates of the corresponding error terms. These error estimates take one of two forms depending whether $\left|u^{2}\left(x-x_{0}\right)^{\lambda+2}\right|$ is bounded away from infinity or bounded away from zero.

More recently, in a survey paper [8] Riekstiņš extended Langer's result in the case of real variables, by showing that the condition concerning the behavior of the function $\left(x-x_{0}\right)^{2} g(x)$ at $x_{0}$ can be eased from analyticity to

$$
\left(x-x_{0}\right)^{2} g(x)=a+O\left\{\left(x-x_{0}\right)^{\alpha}\right\} \quad\left(x \rightarrow x_{0}\right),
$$

where $a$ and $\alpha$ are constants, $\alpha$ being positive.

One of the purposes of the present paper is to show that the conditions on $f(x)$ and $g(x)$ given by Langer and Riekstins can be further weakened. The primary purpose, however, is to supply strict and realistic error bounds for the known asymptotic solutions, both for real and complex values of $u$ and $x$. The availability of error bounds is of obvious computational value. Equally important, however, is the theoretical consideration that by examining the asymptotic behavior of the bounds as $|u| \rightarrow \infty$, we can cope-in an almost trivial manner-with a considerably enlarged class of differential equations of the form (1.01) in which $f(x)$ and $g(x)$ are replaced by functions $f(u, x)$ and $g(u, x)$ that depend on $u$ as well as $x$. Furthermore, the bounds facilitate exploration of the uniformity of the approximate solutions with respect to other parameters that may be present in the differential equation, for example $\lambda$. These extensions greatly increase the scope of the asymptotic theory. The analysis that we shall give draws heavily on results given in the writer's recent book [5], and it will be assumed that the reader has some familiarity with this work.

The case $\lambda<-2$, for which equation (1.01) possesses an irregular singularity at $x=x_{0}$, and the case $\lambda=-2$, for which the singularity is regular, were excluded by Langer and Riekstinš, and will also be excluded in the present investigation. This is because uniform asymptotic approximations in terms of elementary functions, complete with error bounds, have been given for these cases in [5, pp. 203-205 and 220-224]. Other cases for which error bounds have been published include $\lambda=0$ with $g(x)$ continuous at $x_{0}, \lambda=1$ with $g(x)$ continuous at $x_{0}$, and $\lambda=-1$ with $\left(x-x_{0}\right)^{2} g(x)$ analytic at $x_{0}$; see [5, Chapters 6,11 and 12, respectively]. Also, error bounds for $\lambda=2$ with $g(x)$ 
continuous at $x_{0}$ are included as a special case of a theory supplied recently by the present writer for differential equations with two coalescing simple turning points [6].

The results given in the present paper for general values of $\lambda$ specialize into results similar to those just cited for the cases $\lambda=0, \pm 1$ and 2 . The new results also include further cases with $\lambda=0$ and $g(x)$ unbounded as $x \rightarrow x_{0}$ that are important in the calculation of phase shifts in scattering theory; see [5, pp. 208-211 and 449-451]. Thus the present analysis achieves a unification of the error theory of second-order differential equations in regions containing a singularity or turning point that is analogous to the unification in the asymptotic theory achieved by Langer's work. It needs to be emphasized, however, that each of these unifications is confined to the first approximations to the wanted solutions. Except in the cases $\lambda=0, \pm 1$ it is not known how to construct asymptotic expansions for the solutions in descending powers of the large parameter that involve only functions of a single variable; see [4, \$\$2-6].

The paper is arranged as follows. $\$ \S 2-5$ are concerned with real values of $u$ and $x$. In $\$ 2$ the Liouville transformation is applied to convert equation (1.01) into a standard form in which $f(x)$, or more generally $f(u, x)$, is replaced either by $\frac{1}{4}(\lambda+2)^{2} x^{\lambda}$ or by $-\frac{1}{4}(\lambda+2)^{2} x^{\lambda}$, these alternatives being designated Cases I and II, respectively. In $\$ 3$ we state and prove a theorem for Case I which gives approximations to the solutions of the transformed differential equation in terms of modified Bessel functions, complete with error bounds. In $\$ 4$ the uniform asymptotic nature of these approximations is demonstrated by an investigation of the behavior of the error bounds as $u \rightarrow \infty$. For Case II, a similar theorem and asymptotic investigation appear in $\$ 5$; in this case the approximants involve ordinary Bessel functions instead of modified ones. The concluding section, $\S 6$, supplies the corresponding theory for complex variables.

Of course the cases of real $u$ and $x$ treated in $\$ \S 2-5$ can also be regarded as special cases of the analysis given in $\$ 6$ for complex variables. Separate treatments of the real cases are desirable, however, because weaker, nonanalytic, assumptions can then be made concerning the functions $f(u, x)$ and $g(u, x)$, and simpler and sharper error bounds can be found. Furthermore, the methods developed for real variables will be applied in a forthcoming paper [7] to the problem of constructing connection formulas for a transition point of any integer multiplicity.

2. Transformation to standard form: real variables. In accordance with the observations made in $\$ 1$, our starting point is taken to be the differential equation

$$
d^{2} w / d x^{2}=\left\{u^{2}\left(x-x_{0}\right)^{\lambda} f(u, x)+g(u, x) /\left(x-x_{0}\right)^{2}\right\} w
$$


in which $u$ is a positive parameter, $\lambda$ is a number such that $\lambda>-2$, and $f(u, x)$ and $g(u, x)$ are real.(2) This equation has real solutions when $x>x_{0}$, but, in general, these solutions become complex when continued along the real axis through $x_{0}$. Accordingly, we restrict $x$ to the partly closed interval $\left[x_{0}, b\right)$, where the right endpoint $b$ may be finite or $+\infty .{ }^{(3)}$

For $x \in\left[x_{n}, b\right)$ and each value of $u$, we assume that $\partial^{2} f(u, x) / \partial x^{2}$ and $g(u, x)$ are continuous functions of $x$, and $f(u, x)$ is nonzero. All quantities, including $x_{0}, b$, and $\lambda$, may depend on $u$. Since $f(u, x)$ is nonvanishing in $\left[x_{0}, b\right)$, its sign is constant. In Case I we suppose that this sign is positive, and in Case II that the sign is negative.

To convert (2.01) into a standard form, we apply the Liouville transformation. This means that we introduce new variables $W$ and $\zeta$, related by $W=\dot{x}^{-1 / 2} w$, the dot signifying differentiation with respect to $\zeta$. Then $W$ satisfies

$$
\frac{d^{2} W}{d \zeta^{2}}=\left\{u^{2} \dot{x}^{2}\left(x-x_{0}\right)^{\lambda} f(u, x)+\frac{\dot{x}^{2} g(u, x)}{\left(x-x_{0}\right)^{2}}+\dot{x}^{1 / 2} \frac{d^{2}}{d \zeta^{2}}\left(\dot{x}^{-1 / 2}\right)\right\} W
$$

The relation between $x$ and $\zeta$ is prescribed by setting

$$
\dot{x}^{2}\left(x-x_{0}\right)^{\lambda} f(u, x)= \pm \frac{1}{4}(\lambda+2)^{2} \zeta^{\lambda},
$$

and stipulating that $\zeta=0$ corresponds to $x=x_{0}$. Here, and in what follows, the upper sign pertains to Case I and the lower sign to Case II. Integration of (2.03) yields

$$
\zeta^{(\lambda+2) / 2}=\int_{x_{0}}^{x}\left(t-x_{0}\right)^{\lambda / 2}|f(u, t)|^{1 / 2} d t .
$$

This equation defines a one-to-one continuous relationship between $\zeta$ and $x$. We denote by $\beta$ the value of $\zeta$ corresponding to $x=b$; the value of $\beta$ is positive, and finite or infinite depending whether or not the integral in (2.04) converges as $x \rightarrow b$.

Writing

$$
\hat{f}(u, x)=\left(x-x_{0}\right)^{\lambda}|f(u, x)| / \xi^{\lambda}
$$

and substituting in (2.02) by means of (2.03), we arrive at the required form of

(2) Actually $g(u, x)$ can be complex without any significant complications in the analysis and final results.

(3) When $\lambda$ is an integer, equation (2.01) also has real solutions in the interval $x<x_{0}$. These cases can be accommodated in the present theory by replacing $x$ by $-x$. With the further specialization that $\lambda$ is a nonnegative integer and $f(u, x)$ and $g(u, x) /\left(x-x_{0}\right)^{2}$ are continuous at $x_{0}$, each solution is real on both sides of $x_{0}$. The problem of continuing the approximate solutions through $x_{0}$ in these cases is considered in [7]. 
differential equation, given by

$$
d^{2} W / d \zeta^{2}=\left\{ \pm \frac{1}{4}(\lambda+2)^{2} u^{2} \zeta^{\lambda}+\phi(u, \zeta) / \zeta^{2}\right\} W
$$

in which $W=\hat{f}^{1 / 4}(u, x) w$, and

$$
\phi(u, \zeta)=\frac{(\lambda+2)^{2} \zeta^{2} g(u, x)}{4\left(x-x_{0}\right)^{2} \hat{f}(u, x)}+\zeta^{2} \hat{f}^{-1 / 4}(u, x) \frac{d^{2}}{d \zeta^{2}}\left\{\hat{f}^{1 / 4}(u, x)\right\}
$$

On carrying out the differentiations by use of $(2.03)$, we find that when $\phi(u, \zeta)$ is expressed in terms of the original functions it is given by

$$
\begin{aligned}
\frac{\lambda(\lambda+4)}{16} \pm & \frac{(\lambda+2)^{2}}{4}\left(\frac{\zeta}{x-x_{0}}\right)^{\lambda+2} \\
& \quad \times\left\{\frac{g}{f}-\frac{\lambda(\lambda+4)}{16 f}-\left(x-x_{0}\right) \frac{\lambda f^{\prime}}{8 f^{2}}+\left(x-x_{0}\right)^{2}\left(\frac{f^{\prime \prime}}{4 f^{2}}-\frac{5 f^{\prime 2}}{16 f^{3}}\right)\right\}
\end{aligned}
$$

Here the arguments $u$ and $x$ have been suppressed, and primes denote partial differentiations with respect to $x$.

The nature of the functions $\zeta \equiv \zeta(u, x)$ and $\phi(u, \zeta)$ is indicated by the following lemma, the proof of which is a straightforward generalization of the proof given in [5, p. 399] for the case $\lambda=1$, and therefore omitted.

LEMMA. With the conditions stated in the first two paragraphs of this section, $\zeta /\left(x-x_{0}\right)$ is a positive, twice continuously differentiable function of $x$ in $\left[x_{0}, b\right)$, and $\phi(u, \zeta)$ is continuous in the corresponding $\zeta$-interval $[0, \beta) .(4)$

3. Real variables: Case I. From now on it will be convenient to replace the symbol $\lambda$ by $\mu-2$; thus $\mu$ is a positive number that is independent of $x$, though not necessarily independent of $u$.

By the lemma of $\S 2$ the function $\phi(u, \zeta)$ is continuous on the right at $\zeta=0$. We introduce a number $\nu$ defined by the relation

$$
\frac{1}{4}\left(\mu^{2} \nu^{2}-1\right)=\phi(u, 0),
$$

and a function $\psi(u, \zeta)$ defined by

$$
\phi(u, \zeta) / \zeta^{2}=\left(\mu^{2} \nu^{2}-1\right) / 4 \zeta^{2}+\psi(u, \zeta) / \zeta .
$$

Clearly $\psi(u, \zeta)$ is a continuous function of $\zeta$ in $(0, \beta)$, and $\psi(u, \zeta)=o(1 / \zeta)$ as $\zeta \rightarrow 0+$. With these substitutions the standard form of differential equation for Case I becomes

(4) At the left endpoints of the intervals, differentiability and continuity apply only on the right. 


$$
\frac{d^{2} W}{d \zeta^{2}}=\left\{\frac{\mu^{2} u^{2}}{4} \zeta^{\mu-2}+\frac{\mu^{2} \nu^{2}-1}{4 \zeta^{2}}+\frac{\psi(u, \zeta)}{\zeta}\right\} W
$$

compare (2.05). The strategy for solving (3.02) will be to approximate the solutions by those of the equation

$$
\frac{d^{2} W}{d \zeta^{2}}=\left(\frac{\mu^{2} u^{2}}{4} \zeta^{\mu-2}+\frac{\mu^{2} \nu^{2}-1}{4 \zeta^{2}}\right) W .
$$

Exact solutions of (3.03) are $\zeta^{1 / 2} I_{p}\left(u \zeta^{\mu / 2}\right)$ and $\zeta^{1 / 2} K_{\nu}\left(u \zeta^{\mu / 2}\right)$, where $I_{v}$ and $K_{\nu}$ denote the modified Bessel functions in the usual notation. In the present paper, we confine attention to nonnegative real values of the order $\nu$. Referring to (3.01) we see that this implies that $\phi(u, 0)>-4$. Cases in which $\phi(u, 0)<-\frac{1}{4}$ could be treated by analogous methods using modified (or unmodified) Bessel functions of purely imaginary order, but details will not be pursued in the present investigation.

For reference, we quote here some pertinent well-known properties of modified Bessel functions of nonnegative order and positive argument, given by

$$
\begin{array}{ll}
\text { (3.04) } I_{\nu}(x) \sim e^{x} /(2 \pi x)^{1 / 2}, \quad K_{\nu}(x) \sim(\pi / 2 x)^{1 / 2} e^{-x} & (x \rightarrow+\infty), \\
\text { (3.05) } I_{\nu}(x) \sim\left(\frac{1}{2} x\right)^{y} / \Gamma(\nu+1), \quad K_{\nu}(x) \sim \Gamma(\nu) / 2\left(\frac{1}{2} x\right)^{\nu} & (x \rightarrow 0+, \nu>0), \\
\text { (3.06) } I_{0}(x)=1+\frac{1}{4} x^{2}+O\left(x^{4}\right), \quad K_{0}(x) \sim \ln \left(x^{-1}\right) & (x \rightarrow 0+) .
\end{array}
$$

Each of these relations may be differentiated with respect to $x$. For fixed $\nu$, $I_{p}(x)$ is positive and strictly increasing in the interval $0<x<\infty$, and $K_{p}(x)$ is positive and strictly decreasing in the same interval. Lastly, the Wronskian relation satisfied by the modified Bessel functions is given by

$$
W\left\{K_{\nu}(x), I_{\nu}(x)\right\}=1 / x .
$$

Before stating the main result of the present section, we shall also need to introduce a balancing function $\Omega_{\nu}(x) .\left(^{5}\right)$ This is any conveniently chosen function of $x$ that is continuous and positive in the interval $0<x<\infty$, and has the properties

$$
\begin{aligned}
\Omega_{\nu}(x)=O(x) & (x \rightarrow+\infty), \\
\Omega_{\nu}(x)=O(1) & (x \rightarrow 0+, \nu>0), \\
\ln \left(x^{-1}\right) \Omega_{0}(x)=O(1) & (x \rightarrow 0+) .
\end{aligned}
$$

(5) The significance of the name is explained in Remark (i) at the end of this section. 
Subject to these conditions the choice of $\Omega_{v}(x)$ is at our disposal. Next, we define

$$
l_{\nu}=\sup _{x \in(0, \infty)}\left\{2 \Omega_{\nu}(x) I_{\nu}(x) K_{\nu}(x)\right\} .
$$

From (3.04), (3.05) and (3.06) it is immediately seen that conditions (3.08) and (3.09) are precisely those needed to ensure that $l_{\text {, }}$ is finite. One possible choice for the balancing function is, in fact, $\Omega_{v}(x)=1 /\left\{2 I_{v}(x) K_{p}(x)\right\}$, and in this event obviously $l_{v}=1$.

THEOREM 1. In the differential equation (3.02), let $u$ be a positive parameter and $\mu$ and $\nu$ numbers that are independent of $x$ and satisfy $\mu>0$ and $\nu>0$. Assume also that for each value of $u$ the function $\psi(u, \zeta)$ is continuous in a finite or infinite interval $0<\zeta<\beta$, and denote

$$
H(u, \zeta)=\frac{1}{\mu} \int \frac{\psi(u, \zeta)}{\Omega_{v}\left(u \zeta^{\mu / 2}\right)} d \zeta .
$$

Then in $(0, \beta)$ equation (3.02) has solutions $W_{1}(u, \zeta)$ and $W_{2}(u, \zeta)$ that are twice continuously differentiable functions of $\zeta$, and have the forms

$$
\begin{aligned}
& W_{1}(u, \zeta)=\zeta^{1 / 2}\left\{I_{\nu}\left(u \zeta^{\mu / 2}\right)+\varepsilon_{1}(u, \zeta)\right\}, \\
& W_{2}(u, \zeta)=\zeta^{1 / 2}\left\{K_{\nu}\left(u \zeta^{\mu / 2}\right)+\varepsilon_{2}(u, \zeta)\right\}
\end{aligned}
$$

where

$$
\begin{gathered}
\left|\varepsilon_{1}(u, \zeta)\right| \leqslant I_{\nu}\left(u \zeta^{\mu / 2}\right)\left[\exp \left\{l_{\nu} \mho_{0, \zeta}(H)\right\}-1\right], \\
\left|\frac{\partial \varepsilon_{1}(u, \zeta)}{\partial \zeta}\right| \leqslant \frac{\mu}{2 \zeta K_{\nu}\left(u \zeta^{\mu / 2}\right)}\left[\exp \left\{l_{\nu} \mho_{0, \zeta}(H)\right\}-1\right], \\
\left|\varepsilon_{2}(u, \zeta)\right| \leqslant K_{\nu}\left(u \zeta^{\mu / 2}\right)\left[\exp \left\{l_{\nu} \mho_{\zeta, \beta}(H)\right\}-1\right] \\
\left|\frac{\partial \varepsilon_{2}(u, \zeta)}{\partial \zeta}\right| \leqslant \frac{\mu}{2 \zeta I_{\nu}\left(u \zeta^{\mu / 2}\right)}\left[\exp \left\{l_{\nu} \mho_{\zeta, \beta}(H)\right\}-1\right],
\end{gathered}
$$

provided that these bounds are finite.

In this result $\checkmark$ denotes the variational operator defined in [5, pp. 27-29]; thus, for example,

$$
\mho_{0,5}(H)=\frac{1}{\mu} \int_{0}^{\zeta} \frac{|\psi(u, v)|}{\Omega_{\nu}\left(u v^{\mu / 2}\right)} d v
$$


Moreover, in conformity with this reference, we call $H(u, \zeta)$ the error-control function for the present differential equation. In definition (3.11) the choice of integration constant is immaterial, because the bounds (3.13) through (3.16) involve only the variation of $\boldsymbol{H}$.

To establish (3.13) and (3.14) we begin by substituting the first of (3.12) in (3.02). After subtracting equation (3.03) satisfied by $\zeta^{1 / 2} I_{,}\left(u \zeta^{\mu / 2}\right)$, we arrive at the following inhomogeneous differential equation for the product of $\zeta^{1 / 2}$ and the error term $\varepsilon_{1}(u, \zeta)$ :

$$
\begin{gathered}
\frac{d^{2}}{d \zeta^{2}}\left\{\zeta^{1 / 2} \varepsilon_{1}(u, \zeta)\right\}-\left(\frac{\mu^{2} u^{2}}{4} \zeta^{\mu-2}+\frac{\mu^{2} \nu^{2}-1}{4 \zeta^{2}}\right)\left\{\zeta^{1 / 2} \varepsilon_{1}(u, \zeta)\right\} \\
=\frac{\psi(u, \zeta)}{\zeta}\left\{\zeta^{1 / 2} I_{\nu}\left(u \zeta^{\mu / 2}\right)+\zeta^{1 / 2} \varepsilon_{1}(u, \zeta)\right\} .
\end{gathered}
$$

Then applying the method of variation of parameters and referring to the Wronskian (3.07), we construct an equivalent Volterra integral equation

$$
\varepsilon_{1}(u, \zeta)=\frac{2}{\mu} \int_{0}^{\zeta} K(\zeta, v) \psi(u, v)\left\{I_{v}\left(u v^{\mu / 2}\right)+\varepsilon_{1}(u, v)\right\} d v,
$$

in which

$$
\mathrm{K}(\zeta, v)=I_{v}\left(u \zeta^{\mu / 2}\right) K_{\nu}\left(u v^{\mu / 2}\right)-K_{\nu}\left(u \zeta^{\mu / 2}\right) I_{v}\left(u v^{\mu / 2}\right) .
$$

When $0<v \leqslant \zeta$ the monotonicity properties of the modified Bessel functions quoted above show that

$$
I_{v}\left(u v^{\mu / 2}\right) \leqslant I_{v}\left(u \zeta^{\mu / 2}\right), \quad K_{v}\left(u \zeta^{\mu / 2}\right)<K_{v}\left(u v^{\mu / 2}\right) .
$$

Hence

$$
0 \leqslant K(\zeta, v)<I_{\nu}\left(u \zeta^{\mu / 2}\right) K_{p}\left(u v^{\mu / 2}\right) \quad(0<v<\zeta) .
$$

Next, differentiation of (3.18) gives

$$
\frac{\partial K(\zeta, v)}{\partial \zeta}=\frac{1}{2} \mu u \zeta^{(\mu-2) / 2}\left\{I_{\nu}^{\prime}\left(u \zeta^{\mu / 2}\right) K_{\nu}\left(u v^{\mu / 2}\right)-K_{v}^{\prime}\left(u \zeta^{\mu / 2}\right) I_{v}\left(u v^{\mu / 2}\right)\right\} .
$$

Applying (3.19) and bearing in mind that $I_{v}^{\prime}$ is positive and $K_{\nu}^{\prime}$ is negative, we see that

$$
\begin{aligned}
\left|\frac{\partial K(\zeta, v)}{\partial \zeta}\right| \leqslant \frac{1}{2} \mu u \zeta^{(\mu-2) / 2}\{ & I_{\nu}^{\prime}\left(u \zeta^{\mu / 2}\right) K_{\nu}\left(u v^{\mu / 2}\right) \\
& \left.-K_{\nu}^{\prime}\left(u \zeta^{\mu / 2}\right) I_{\nu}\left(u v^{\mu / 2}\right) \frac{K_{\nu}\left(u v^{\mu / 2}\right) I_{\nu}\left(u \zeta^{\mu / 2}\right)}{K_{\nu}\left(u \zeta^{\mu / 2}\right) I_{\nu}\left(u v^{\mu / 2}\right)}\right\},
\end{aligned}
$$


and hence, by use of (3.07), obtain

$$
\left|\frac{\partial K(\zeta, v)}{\partial \zeta}\right| \leqslant \frac{\mu}{2 \zeta} \frac{K_{\nu}\left(u v^{\mu / 2}\right)}{K_{\nu}\left(u \zeta^{\mu / 2}\right)} \quad(0<v \leqslant \zeta) .
$$

Having bounded the kernel and its $\zeta$-derivative, we can solve the integral equation (3.17) by the standard procedure of successive approximation, $(6)$ and on using the definitions (3.10) and (3.11) we arrive at the desired bounds (3.13) and (3.14). The proof of (3.15) and (3.16) is similar.

REMARKS. (i) The name "balancing function" is suggested by the following consideration. If $\Omega_{v}(x)$ is changed, then both the quantity $l_{\nu}$ defined by (3.10) and the total variation $\Upsilon_{0, \beta}(H)$ of the error-control function (3.11) are affected. Moreover, it is apparent that when one of these quantities increases the other is likely to decrease. To minimize the error bounds (3.13) to (3.16) we need to choose $\Omega_{v}(x)$ in such a way that the best balance is struck between $l_{\nu}$ and $\mho_{0, \beta}(H)$ in forming the product of these quantities. ( $(7)$

(ii) The inequality (3.13) furnishes direct information concerning the relative error $\varepsilon_{1}(u, \zeta) / I_{\nu}\left(u \zeta^{\mu / 2}\right)$ in the first of the approximations (3.12). On the other hand, inequality (3.14) does not supply direct information concerning the ratio of $\partial \varepsilon_{1}(u, \zeta) / \partial \zeta$ to $d I_{\nu}\left(u \zeta^{\mu / 2}\right) / d \zeta$, that is, $\frac{1}{2} \mu u \zeta^{(\mu-2) / 2} I_{\nu}^{\prime}\left(u \zeta^{\mu / 2}\right)$. Meaningful information is available indirectly, however, because the differentiated forms of (3.04), (3.05) and (3.06) show that, except when $\nu=0$, the ratio of $1 / K_{\nu}(x)$ to $x I_{\nu}^{\prime}(x)$ is finite and bounded throughout the interval $x \in(0, \infty)$. The apparent failure in the case $\nu=0$ is attributable to the presence of a zero in $I_{0}^{\prime}(x)$ at $x=0$.

Similar observations apply to (3.16), except that the case $\nu=0$ is no longer special. This is because the ratio of $1 / I_{\nu}(x)$ to $x K_{\nu}^{\prime}(x)$ is finite and bounded throughout $x \in(0, \infty)$ for each value of $\nu$, including zero.

(iii) The condition that $\psi(u, \zeta)$ be continuous when $\zeta \in(0, \beta)$ is not essential. From the conditions given on p. 218 of [5] it can be seen that relations (3.12) to (3.16) still apply when $\psi(u, \zeta)$ has a finite number of discontinuities in $(0, \beta)$, provided that $\mho_{0, \beta}(H)$ is finite. The only change in Theorem 1 is that $\partial^{2} W_{1} / \partial \zeta^{2}$ and $\partial^{2} W_{2} / \partial \zeta^{2}$ are discontinuous at the discontinuities of $\psi(u, \zeta)$.

(iv) In the case $\mu=1$, inequality (3.21) could be applied to the analysis of [5, pp. 441-442], to derive bounds similar to (3.14) and (3.16) for the derivatives of the error terms associated with the series expansions of the solutions. These bounds are simpler than those given in [5].

(๑) For example, by application of Theorem 10.2 on p. 220 of [5].

(7) "Weight function" could be used in place of "balancing function", but this term is used in a different context in $\$ \$ 5$ and 6. 
4. Asymptotic properties of the approximute ns. In order that the bounds (3.13) and (3.15) for the relative errors $i$ approximations to the solutions $W_{1}(u, \zeta)$ and $W_{2}(u, \zeta)$ are finite, it suft. that the error-control function $H(u, \zeta)$ defined by $(3.11)$ is of bounded variation in the interval $(0, \beta)$. From condition (3.08) it is clear that by proper choice of the balancing function $\Omega_{v}(x)$ we can arrange for the variation of $H(u, \zeta)$ to converge at $\zeta=\beta$, provided that $\zeta^{-\mu / 2}|\psi(u, \zeta)|$ is integrable at $\beta$. Similarly, at the other endpoint $\zeta=0$ the variation of $H(u, \zeta)$ can be made to converge as long as $|\psi(u, \zeta)|$ is integrable when $\nu>0$, or $|\psi(u, \zeta) \ln \zeta|$ is integrable when $\nu=0$.

What is the asymptotic behavior of the error bounds as the parameter $u$ tends to infinity? The answer obviously depends on the way in which the function $\psi(u, \zeta)$ involves the parameter $u$. For illustration, we consider an example in which $\beta=\infty$, and each of $\mu, \nu$, and $\psi(u, \zeta)=\psi(\zeta)$ is independent of $u$. Other cases may be attacked along similar lines.

ExaMPLE. Assume that:

(i) $\psi(\zeta)$ is continuous in $(0, \infty)$.

(ii) $\zeta^{-\mu / 2}|\psi(\zeta)|$ is integrable at $\zeta=+\infty$.

(iii) $\psi(\zeta)=O\left(\zeta^{\rho-1}\right)$ as $\zeta \rightarrow 0+$, where $\rho$ is a positive constant.

We first observe that these conditions ensure that $\psi(\zeta)$ meets all the conditions given in $\$ 3$ and the first paragraph in the present section. An admissible choice of $\Omega_{\nu}(x)$ is furnished by

$$
\Omega_{\nu}(x)=1+x(\nu>0), \quad \Omega_{0}(x)=(1+x) / \ln \left(e+x^{-1}\right),
$$

where $e$ is the base of natural logarithms. In consequence of condition (iii) there exists a constant $A$ such that $|\psi(\zeta)|<A \zeta^{p-1}(0<\zeta<1)$. Hence in the case $\nu>0$ we have

$$
\mho_{0,1}(H)=\frac{1}{\mu} \int_{0}^{1} \frac{|\psi(v)|}{1+u v^{\mu / 2}} d v<\frac{A}{\mu} \int_{0}^{1} \frac{v^{\rho-1} d v}{1+u v^{\mu / 2}}=\frac{2 A}{\mu^{2} u^{2 \rho / \mu}} \int_{0}^{u} \frac{\tau^{(2 \rho / \mu)-1}}{1+\tau} d \tau .
$$

The last quantity is estimated by

$$
\begin{gathered}
O\left(u^{-1}\right) \quad(\mu<2 \rho), \quad O\left(u^{-1} \ln u\right) \quad(\mu=2 \rho), \text { or } \\
O\left(u^{-2 \rho / \mu}\right) \quad(\mu>2 \rho) .
\end{gathered}
$$

Next,

$$
\mho_{1, \infty}(H)=\frac{1}{\mu} \int_{1}^{\infty} \frac{|\psi(v)|}{1+u v^{\mu / 2}} d v \leqslant \frac{1}{\mu u} \int_{1}^{\infty} \frac{|\psi(v)|}{v^{\mu / 2}} d v=O\left(\frac{1}{u}\right) ;
$$

compare condition (ii). Adding this estimate to that for $\Upsilon_{0,1}(H)$ given by (4.01), we see that $\mho_{0 . \infty}(H)$, also, is estimated by (4.01). 
When $\nu=0$, the analysis is modified by introduction of the factor $\ln \left(e+u^{-1} v^{-\mu / 2}\right)$ in the integrals for $\mho_{0,1}(H)$ and $\mho_{1, \infty}(H)$. It is easy to verify, however, that the final estimates (4.01) continue to apply to $\mho_{0, \infty}(H)$.

Bearing in mind that the quantity $l_{v}$ defined by (3.10) is independent of $u$, we see from (3.13) and (3.15) that the relative errors $\varepsilon_{1}(u, \zeta) / I_{v}\left(u \zeta^{\mu / 2}\right)$ and $\varepsilon_{2}(u, \zeta) / K_{\nu}\left(u \zeta^{\mu / 2}\right)$ in the approximations (3.12) each have the estimates (4.01) as $u \rightarrow \infty$, uniformly with respect to $\zeta \in(0, \infty)$. Moreover, the same estimates also hold for $\zeta K_{\nu}\left(u \zeta^{\mu / 2}\right) \partial \varepsilon_{1}(u, \zeta) / \partial \zeta$ and $\zeta I_{\nu}\left(u \zeta^{\mu / 2}\right) \partial \varepsilon_{2}(u, \zeta) / \partial \zeta .\left(^{(8)}\right.$ These are the required asymptotic results for the present example.

5. Real variables: Case II. The standard form of differential equation for Case II is given by

$$
\frac{d^{2} W}{d \zeta^{2}}=\left\{-\frac{\mu^{2} u^{2}}{4} \zeta^{\mu-2}+\frac{\mu^{2} \nu^{2}-1}{4 \zeta^{2}}+\frac{\psi(u, \zeta)}{\zeta}\right\} W
$$

The approximating equation is taken to be

$$
\frac{d^{2} W}{d \zeta^{2}}=\left(-\frac{\mu^{2} u^{2}}{4} \zeta^{\mu-2}+\frac{\mu^{2} \nu^{2}-1}{4 \zeta^{2}}\right) W
$$

with solutions $\zeta^{1 / 2} J_{\nu}\left(u \zeta^{\mu / 2}\right)$ and $\zeta^{1 / 2} Y_{\nu}\left(u \zeta^{\mu / 2}\right)$.

For nonnegative values of the order $\nu$ and positive values of the argument $x$, relevant properties of the Bessel functions are given, for example, in [5, pp. 436-438]. And exactly as in this reference we introduce auxiliary weight, modulus, and phase functions $E_{\nu}(x), M_{\nu}(x)$, and $\theta_{\nu}(x)$, respectively, such that

$$
J_{\nu}(x)=E_{\nu}^{-1}(x) M_{\nu}(x) \cos \theta_{\nu}(x), \quad Y_{\nu}(x)=E_{\nu}(x) M_{\nu}(x) \sin \theta_{\nu}(x) .
$$

We shall also need to introduce corresponding modulus and phase functions for the derivatives; thus

$$
J_{\nu}^{\prime}(x)=E_{\nu}^{-1}(x) N_{\nu}(x) \cos \omega_{\nu}(x), \quad Y_{\nu}^{\prime}(x)=E_{\nu}(x) N_{\nu}(x) \sin \omega_{\nu}(x),
$$

with the same weight function $E_{\nu}(x)$ as in (5.03).

The balancing function $\Omega_{\nu}(x)$ and error-control function $H(u, \zeta)$ are defined exactly as in 83 above. Auxiliary positive constants that will be needed are defined by

$$
\begin{gathered}
l_{\nu, 0}=\sup \left\{\pi \Omega_{\nu}(x) M_{\nu}^{2}(x)\right\}, \quad l_{\nu, 1}=\sup \left\{\pi \Omega_{\nu}(x)\left|J_{\nu}(x)\right| E_{\nu}(x) M_{\nu}(x)\right\}, \\
l_{\nu, 2}=\sup \left\{\pi \Omega_{\nu}(x)\left|Y_{\nu}(x)\right| E_{\nu}^{-1}(x) M_{\nu}(x)\right\}
\end{gathered}
$$

(8) Compare Remark (ii) at the end of 83. 
each supremum being taken over the $x$-interval $(0, \infty)$. It is easily verified that all three suprema are finite; moreover, $l_{v, 1} \leqslant l_{v, 0}$ and $l_{v, 2}<l_{v, 0}$.

THEOREM 2. Let $u$ be a positive parameter and $\mu$ and $\nu$ be numbers that are independent of $x$ and satisfy $\mu>0$ and $\nu>0$. Assume also that for each value of $u$ the function $\psi(u, \zeta)$ is continuous in a finite or infinite interval $0<\zeta<\beta$. Then in this interval the differential equation $(5.01)$ has solutions $W_{1}(u, \zeta)$ and $W_{2}(u, \zeta)$ that are twice continuously differentiable functions of $\zeta$, and have the forms

where

$$
\begin{aligned}
& W_{1}(u, \zeta)=\zeta^{1 / 2}\left\{J_{\nu}\left(u \zeta^{\mu / 2}\right)+\varepsilon_{1}(u, \zeta)\right\}, \\
& W_{2}(u, \zeta)=\zeta^{1 / 2}\left\{Y_{\nu}\left(u \zeta^{\mu / 2}\right)+\varepsilon_{2}(u, \zeta)\right\},
\end{aligned}
$$

$$
\begin{aligned}
& \left|\varepsilon_{1}(u, \zeta)\right| \leqslant\left(l_{v, 1} / l_{v, 0}\right) E_{\nu}^{-1}\left(u \zeta^{\mu / 2}\right) M_{v}\left(u \zeta^{\mu / 2}\right)\left[\exp \left\{l_{v, 0} \sigma_{v, \zeta}(H)\right\}-1\right], \\
& \left|\partial \varepsilon_{1}(u, \zeta) / \partial \zeta\right| \leqslant \frac{1}{2} \mu\left(l_{v, 1} / l_{\nu, 0}\right) u \zeta^{(\mu-2) / 2} E_{\nu}^{-1}\left(u \zeta^{\mu / 2}\right) N_{v}\left(u \zeta^{\mu / 2}\right) \\
& \times\left[\exp \left\{l_{\nu, 0} \mho_{0,5}(H)\right\}-1\right], \\
& \left|\varepsilon_{2}(u, \zeta)\right| \leqslant\left(l_{v, 2} / l_{\nu, 0}\right) E_{\nu}\left(u \zeta^{\mu / 2}\right) M_{\nu}\left(u \zeta^{\mu / 2}\right) \times\left[\exp \left\{l_{v, 0} \mho_{\zeta, \beta}(H)\right\}-1\right], \\
& \left|\partial \varepsilon_{2}(u, \zeta) / \partial \zeta\right| \leqslant \frac{1}{2} \mu\left(l_{v, 2} / l_{v, 0}\right) u \zeta^{(\mu-2) / 2} E_{v}\left(u \zeta^{\mu / 2}\right) N_{v}\left(u \zeta^{\mu / 2}\right) \\
& \times\left[\exp \left\{l_{v, 0} \mho_{\zeta, \beta}(H)\right\}-1\right],
\end{aligned}
$$

provided that these bounds are finite.

The proof of this theorem is similar to that of Theorem 1, and it is unnecessary to record details.

Similar observations to those made in $\$ \$ 3$ and 4 also apply in the present case. Thus if $\psi(u, \zeta) \equiv \psi(\zeta)$ is independent of $u$, and satisfies the conditions of the example given in $\$ 4$, then both

$$
\varepsilon_{1}(u, \zeta) /\left\{E_{\nu}^{-1}\left(u \zeta^{\mu / 2}\right) M_{\nu}\left(u \zeta^{\mu / 2}\right)\right\}, \quad \varepsilon_{2}(u, \zeta) /\left\{E_{\nu}\left(u \zeta^{\mu / 2}\right) M_{\nu}\left(u \zeta^{\mu / 2}\right)\right\},
$$

are estimated by $(4.01)$ as $u \rightarrow \infty$, uniformly with respect to $\zeta \in(0, \infty)$. Similarly for the derivatives. It may be noted that this particular example was also treated by Riekstings, and our results resemble those given on p. 73 of [8].

6. Complex parameter and argument. In this concluding section we consider equation (3.02), that is,

$$
\frac{d^{2} W}{d \zeta^{2}}=\left\{\frac{\mu^{2} u^{2}}{4} \zeta^{\mu-2}+\frac{\mu^{2} \nu^{2}-1}{4 \zeta^{2}}+\frac{\psi(u, \zeta)}{\zeta}\right\} W
$$


for complex values of $u$ and $\zeta$. We continue to suppose that $\mu$ is real and positive, and $\nu$ is real and nonnegative. We assume that for each value of $u$ there is an open domain $\Delta(u)$, which contains $\zeta=0$ and may be unbounded, and $\psi(u, \zeta)$ is holomorphic in $\Delta(u)$ except possibly at $\zeta=0$. The domain $\Delta(u)$ need not be confined to a single Riemann sheet.

Relevant properties of the modified Bessel functions for real orders and complex arguments are given in [5, pp. 453-454], and exactly as on pp. 454-457 of this reference we introduce real weight, modulus, and phase functions $\mathbb{F}_{\nu}(z), \mathfrak{R}_{\nu}(z)$, and $\vartheta_{\nu}(z)$, respectively, such that when $|\mathrm{ph} z| \leqslant \frac{1}{2} \pi,\left({ }^{9}\right)$

$$
\left|I_{\nu}(z)\right|=E_{\nu}(z) \mathbb{R}_{\nu}(z) \cos \vartheta_{\nu}(z), \quad\left|K_{\nu}(z)\right|=E_{\nu}^{-1}(z) \mathbb{R}_{\nu}(z) \sin \vartheta_{\nu}(z) .
$$

With the same weight function $E_{\nu}(z)$ we also introduce corresponding modulus and phase functions for the derivatives, given by

$$
\left|I_{\nu}^{\prime}(z)\right| \stackrel{\dot{=}}{=} \xi_{\nu}(z) \mathfrak{R}_{\nu}(z) \cos \varpi_{\nu}(z), \quad\left|K_{\nu}^{\prime}(z)\right|=\varepsilon_{\nu}^{-1}(z) \mathfrak{R}_{\nu}(z) \sin \varpi_{\nu}(z),
$$

again valid when $|\operatorname{ph} z| \leqslant \frac{1}{2} \pi$.

Next, the balancing function $\Omega_{v}(z)$ is defined to be a real or complex continuous function of $z$, not necessarily analytic, which is nonvanishing in the sector $|\operatorname{ph} z| \leqslant \frac{1}{2} \pi$, except possibly at $z=0$, and has the following properties in this sector:

$$
\begin{aligned}
\Omega_{\nu}(z) & =O(z) & & (|z| \rightarrow \infty), \\
\Omega_{\nu}(z) & =O(1) & & (|z| \rightarrow 0, \nu>0), \\
\ln \left(z^{-1}\right) \Omega_{0}(z) & =O(1) & & (|z| \rightarrow 0),
\end{aligned}
$$

each 0 -term being uniform with respect to $\mathrm{ph} z$. The auxiliary positive constants needed in the present case are defined by

$$
\begin{gathered}
L_{\nu, 0}=\sup \left\{2\left|\Omega_{\nu}(z)\right| \mathfrak{M}_{\nu}^{2}(z)\right\}, \quad L_{\nu, 1}=\sup \left\{2\left|\Omega_{\nu}(z) I_{\nu}(z)\right| ほ_{\nu}^{-1}(z) \mathbb{M}_{\nu}(z)\right\}, \\
L_{\nu, 2}=\sup \left\{2\left|\Omega_{\nu}(z) K_{\nu}(z)\right| \uplus_{\nu}(z) \mathbb{R}_{\nu}(z)\right\} .
\end{gathered}
$$

Each supremum is taken over the sector $|\operatorname{ph} z| \leqslant \frac{1}{2} \pi$, and is easily seen to be finite.

Lastly, we again define

$$
H(u, \zeta)=\frac{1}{\mu} \int \frac{\psi(u, \zeta)}{\Omega_{\nu}\left(u \zeta^{\mu / 2}\right)} d \zeta,
$$

(9) Outside the sector $|\operatorname{ph} z|<\frac{1}{2} \pi$ the auxiliary functions are undefined. 
with any convenient choice of the constant of integration.

THEOREM 3. With the conditions and definitions of the present section, the differential equation (6.01) has, for each value of $u$, solutions $W_{1}(u, \zeta)$ and $W_{2}(u, \zeta)$ which are holomorphic in $\Delta(u)$, save at $\zeta=0$, and have the forms

$$
\begin{aligned}
& W_{1}(u, \zeta)=\zeta^{1 / 2}\left\{I_{\nu}\left(u \zeta^{\mu / 2}\right)+\varepsilon_{1}(u, \zeta)\right\}, \\
& W_{2}(u, \zeta)=\zeta^{1 / 2}\left\{K_{\nu}\left(u \zeta^{\mu / 2}\right)+\varepsilon_{2}(u, \zeta)\right\},
\end{aligned}
$$

where

$$
\begin{aligned}
\left|\varepsilon_{1}(u, \zeta)\right| \leqslant\left(L_{\nu, 1} / L_{\nu, 0}\right) E_{\nu}\left(u \zeta^{\mu / 2}\right) \mathbb{M}_{\nu}\left(u \zeta^{\mu / 2}\right)\left[\exp \left\{L_{\nu, 0} \mho_{0, \zeta}(H)\right\}-1\right] & \\
\left|\partial \varepsilon_{1}(u, \zeta) / \partial \zeta\right| \leqslant & \frac{1}{2} \mu\left(L_{\nu, 1} / L_{\nu, 0}\right)\left|u \zeta^{(\mu-2) / 2}\right| \Theta_{\nu}\left(u \zeta^{\mu / 2}\right) \Re_{\nu}\left(u \zeta^{\mu / 2}\right) \\
& \times\left[\exp \left\{L_{\nu, 0} \mho_{0, \zeta}(H)\right\}-1\right]
\end{aligned}
$$

when $\zeta \in \mathbf{Z}_{1}(u)$ (defined below), and

$$
\begin{aligned}
\left|\varepsilon_{2}(u, \zeta)\right| \leqslant\left(L_{v, 2} / L_{v, 0}\right) \xi_{\nu}^{-1}\left(u \zeta^{\mu / 2}\right) \mathbb{R}_{\nu}\left(u \zeta^{\mu / 2}\right)\left[\exp \left\{L_{v, 0} \mho_{\zeta, \beta}(H)\right\}-1\right] & \\
\left|\partial \varepsilon_{2}(u, \zeta) / \partial \zeta\right|< & \frac{1}{2} \mu\left(L_{v, 2} / L_{v, 0}\right)\left|u \zeta^{(\mu-2) / 2}\right| \xi_{\nu}^{-1}\left(u \zeta^{\mu / 2}\right) \Re_{\nu}\left(u \zeta^{\mu / 2}\right) \\
& \times\left[\exp \left\{L_{v, 0} \mho_{\zeta, \beta}(H)\right\}-1\right]
\end{aligned}
$$

when $\zeta \in \mathbf{Z}_{\mathbf{2}}(u, \beta)$ (defined below).

In this result the regions of validity $\mathbf{Z}_{1}(u)$ and $\mathbf{Z}_{2}(u, \beta)$ are specified in a similar manner to the case $\mu=1$ treated in $\left[5, p_{.} 457\right]$. Thus we denote by $Z(u)$ the intersection of $\Delta(u)$ and the sector $\left|\operatorname{ph}\left(u^{\mu / 2}\right)\right| \leqslant \frac{1}{2} \pi$. Any branch of $\zeta^{\mu / 2}$ may be employed, provided that it is continuous and used consistently throughout.($\left.{ }^{10}\right)$ Then $Z_{1}(u)$ comprises the set of points $\zeta$ that can be linked to the origin by a path(11) $2_{1}$ lying in $Z(u)$ such that as $v$ passes along $2_{1}$ from 0 to $\zeta$ both $\operatorname{Re}\left(u v^{\mu / 2}\right)$ and $|v|$ are nondecreasing. The other region of validity $\mathbf{Z}_{2}(u, \beta)$ comprises the set of points $\zeta$ that can be linked to an arbitrary point $\beta=\beta(u)$ of $\mathbf{Z}(u)$ (possibly at infinity), by a path $2_{2}$ lying in $\mathbf{Z}(u)$ such that as $v$ passes along $2_{2}$ from $\beta$ to $\zeta$ both $\operatorname{Re}\left(u v^{\mu / 2}\right)$ and $|v|$ are nonincreasing. Other matters to be noted are (a) the argument $u \zeta^{\mu / 2}$ of each function appearing in (6.04) to (6.09) is the branch satisfying $\left|\mathrm{ph}\left(u \zeta^{\mu / 2}\right)\right| \leqslant \frac{1}{2} \pi$; (b) the variations in (6.06) and (6.07) are evaluated along the path $2_{1}$, and the variations in (6.08)

(10) See also Remark (i) below.

(II) Strictly, here, and in the definition of $Z_{2}(u, \beta)$, a "path" means a finite chain of $R_{2}$-arcs in the sense of $[5$, p. 147]. 
and (6.09) are evaluated along $2_{2}$; (c) the solution $W_{2}(u, \zeta)$ depends on the choice of $\beta$.

The proof of Theorem 3 is similar to that of Theorem 1 above and Theorem 9.1 of [5, Chapter 12], and details are omitted.

REMARKS. (i) By employing all possible branches of $\zeta^{\mu / 2}$ in the definition of the region $\mathbf{Z}(u)$, the whole of the original domain $\Delta(u)$ can be covered by a sequence of abutting sectors of angle $2 \pi / \mu$. In each sector, Theorem 3 furnishes a pair of solutions of equation (6.01). The pair differs from sector to sector.

(ii) For large $|u|$ uniform asymptotic properties of the approximate solutions can be investigated by a straightforward extension of the methods indicated in 84.

ACKNOWLEDGMENT. The writer is pleased to acknowledge several suggestions by the referee that improved the presentation of the results.

\section{REFERENCES}

1. R. E. Langer, On the asymptotic solutions of ordinary differential equations, with an application to the Bessel functions of large order, Trans. Amer. Math. Soc. 33 (1931), 23-64.

2. - On the asymptotic solutions of differential equations, with an application to the Bessel functions of large complex order, Trans. Amer. Math. Soc. 34 (1932), 447-480.

3. - On the asymptotic solutions of ordinary differential equations, with reference to the Stokes' phenomenon about a singular point, Trans. Amer. Math. Soc. 37 (1935), 397-416.

4. F. W. J. Olver, The asymptotic solution of linear differential equations of the second order in a domain containing one transition point, Philos. Trans. Roy. Soc. London Ser. A 249 (1956), 65-97. MR 18, 38.

5. - Asymptotics and special functions, Academic Press, New York, 1974.

6. - Second-order linear differential equations with two turning points, Philos. Trans. Roy. Soc. London Ser. A 278 (1975), 137-174.

7. - Connection formulas for second-order differential equations with multiple turning points, SIAM J. Math. Anal. 8 (1977), 127-154.

8. E. Riekstiņ̌, On the method of tabulated functions, Latvijas Valsts Univ. Zinātn. Raksti 20 (1958), 65-86. (Russian) MR 21 \#2095; 22, p. 2545.

Institute for Physical Science and Technology, University of Maryland, Collegb Park, MaryLand 20742

Applied Mathematics Division, National Bureau of Standards, Washington, D.C. 20234 